\title{
Identification of the moving vehicle presence on the bridge with the time-frequency analysis
}

\author{
Ján Kortiš ${ }^{1,{ }^{*}, \text { Luboš } \text { Daniel }^{1} \text { and Michaela Holešová }}{ }^{1}$ \\ ${ }^{1}$ University of Žilina, Faculty of Civil Engineering, Univerzitná 8215/1, 01026 Žilina, Slovakia
}

\begin{abstract}
The article presents the application of common techniques of signal processing as the Wavelet Transformation and Short-time Fourier Transformation to detect the position of the moving vehicle on the bridge. The measured acceleration in the middle of the span of the investigated bridge structure are used for this purpose. The aim of the analysis is to determine the time when the vehicle comes to the bridge and when it leaves. This can be important to know if there is required to investigate free and forced vibration of the bridge separately. It is done for two cases that differ in presence of the obstacle which is positioned on the road in the middle of the span. The obstacle is used to increase the dynamic excitation of the bridge. It is recommended by Slovak standard STN 736209 for measurement of dynamic characteristics of bridges as a source of excitation.
\end{abstract}

\section{Introduction}

The measurement of dynamic characteristics of bridges is an essential practice in the assessment of these structures [1]. The measurement techniques that are used to identify natural frequencies and natural shapes are based on the techniques of operational modal analysis [2]. However, the operational modal analysis is effective technique with high accuracy, it has got a few practical issues. One of the biggest is to find the suitable and sufficiently powerful source of excitation. The vehicle moving through the obstacle on the bridge is a good alternative how to excite the bridge. In this case, the presence of the heavy vehicle on the bridge could influence the results obtained from the measurement [3]. Because of this, there is important to know the position of the vehicle to recognise if the bridge oscillate without the mass of the vehicle or with that and how they differ. For this purpose, there is required to know the time period in the signal while the vehicle is on the bridge and while it is not. The direct detection of the moment when the vehicle comes to the bridge and when it leaves is one possible way. The special measured equipment like steel plates with accelerometers that are located at the beginning and at the end of the bridge was used for this purpose (Fig. 1).

\footnotetext{
*Corresponding author: jan.kortis@fstav.uniza.sk
} 


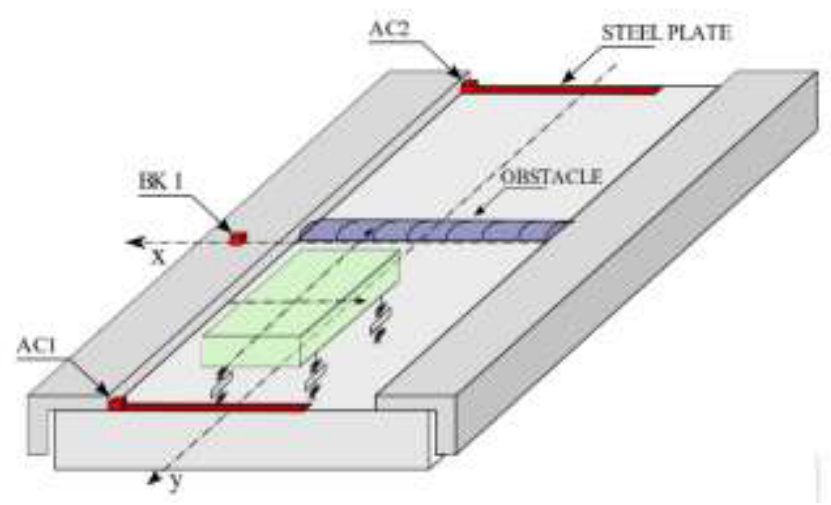

Fig. 1. The scheme of experimental measurement with the location of the steel plates with accelerometers (AC1, AC2), accelerometer BK1 measured response of the bridge.

The measured signals of the accelerometers AC1 and AC2 significantly change when the wheels of the vehicle reach the steel plates. This is used to detect the moment when the vehicle reaches the places where they are positioned (Fig. 2). They give good results but there is a problem that they only give information in time about the moment when the vehicle comes to the bridge and when it leaves. There is no information about the real position of the vehicle on the bridge.

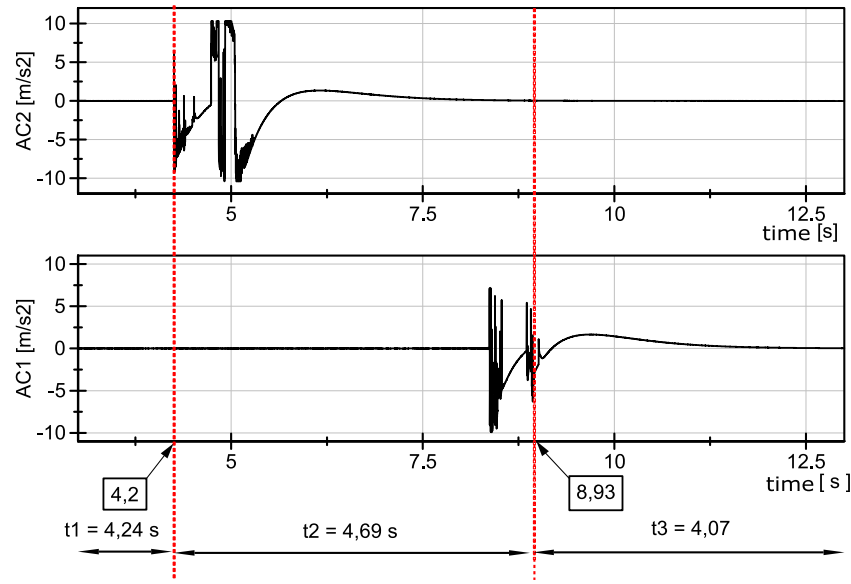

Fig. 2. The identification of the position of the vehicle on the bridge trough the response of the accelerometers AC1 and AC2

The next possible way how to detect the position of the vehicle is to investigate the changes in the frequency spectrum of measured acceleration that arise when the vehicle is moving through the bridge. The Fast Fourier Transformation gives information just about the changes in the frequency domain of the whole signal. In the article, there are presented two different transformations Short Fourier Transformation and Continuous Wavelet Transformation which can show the changes of frequency domain in time [4], [5]. 


\section{Mathematical background of the analysis}

The Fourier Transformation is used to transform the signal from time domain to the frequency domain. There is assumed that the signal has an infinite length and it does not change in time. Nonetheless, the real signals are not infinite and they are nonstationary. Thus, the transformation that is useful for practical purposes desires also the possibility to provide the information about the changes of frequency components of the spectrum with respect to time. Determining the time localization of frequency components of nonstationary signals requires time-frequency transformation such as Short Time Fourier Transformation or Continuous Wavelet Transformation.

Let the Hilbert space

$$
L^{2}(R)=\left\{g: R \rightarrow C, \quad \int_{-\infty}^{\infty}|g(x)|^{2} d x<\infty\right\}
$$

and the scalar product of functions $f, h \in L^{2}(R)$ is

$$
\langle f, h\rangle=\int_{-\infty n}^{\infty} f(x) \overline{h(x)} d x,
$$

where $\overline{h(x)}$ complex conjugation of the function $h(x)$. If the signal represented by a function $f$ of the Hilbert space $L^{2}(R)$, then Short Time Fourier Transform (STFT) of signal $f(t)$ is based on the fact that we analyse this function using of some window function $h \in L^{2}(R)$ with constant length. Then there is computed the Fourier Transformation separately on each shorter segment of a continuous signal $f(t)$, hence STFT is defined as follows

$$
\operatorname{STFT}\{f(t)\}(\omega, \tau)=\int_{-\infty}^{\infty} f(t) \overline{h(t-\tau)} e^{-i \omega t} d t,
$$

Where $\overline{h(t-\tau)}$ complex conjugation of the function $h(t-\tau)$. We used for our computation Gaussian window with length $N=256$.

STFT decomposes (localizes) this function of signals in time-frequency domain $(t . \omega) \subset R \times R$. Spectrogram SPEC $(t, \omega)$ is used for graphical representation of STFT as a complex function. It is the function of two variables identified as a square of modulus of the STFT, i.e.

$$
\operatorname{SPEC}(t, \omega)=|\operatorname{STFT}\{f(t)\}(\omega, \tau)|^{2} .
$$

Although STFT gives information between time and frequency representation of signals, it cannot reach the high resolution in time and frequency. Selecting of a long window allows good frequency resolution, but worse time resolution. Short window leads to a good resolution in time but poor frequency resolution. Therefore, the compromise between precision of the determination of frequency and time have to be find by the optimal width of the window. However, this can cause mistakes if results with high accuracy are required. For example, good resolution helps to detect the moment when vehicle enters the bridge precisely. In this case, Continuous Wavelet Transformation (CWT) seems to be a better alternative. CWT can be used to discover difference of the signal properties at various scales in time [6], [7]. This transformation is suitable for the analysis of continuous signal that considerably varies. It gives good time resolution at higher frequencies.

Let $\psi \subset L^{2}(R)$ a mother (base) wavelet function which is usually $\|\psi(t)\|=1$. 
We define

$$
\psi_{a}(t)=\frac{1}{\sqrt{a}} \psi\left(\frac{t}{a}\right) \text { and } \psi_{a, b}(t) \equiv \psi_{a}(t-b)=\frac{1}{\sqrt{a}} \psi\left(\frac{t-b}{a}\right) .
$$

Continuous wavelet transform (CWT) of a signal $f \in L^{2}(R)$ is

$$
C W T\{f(t)\}(a, b)=\int_{-\infty}^{\infty} f(t) \overline{\psi_{a, b}(t)} d t=\frac{1}{\sqrt{a}} \int_{-\infty}^{\infty} f(t) \overline{\psi\left(\frac{t-b}{a}\right)} d t=\left\langle f, \psi_{a, b}(t)\right\rangle,
$$

Where $\overline{\psi_{a, b}(t)}$ complex conjugation of the function $\psi_{a, b}(t)$. The number $a>0$ is the scaling parameter and the mother wavelet $\psi$ for $a>1$ expands in the time domain that corresponds to a compression in the frequency domain. For $0<a<1$, the wavelet compressed in the time domain that corresponds to expansion in the frequency domain. The number $b \in R$ determines the size of displacement of wavelet in the timeline. This increases the ability to distinguish signals in time. If the signal contains, for example, some discontinuity at the time $t=t_{1}$ then for small values of the parameter $a$ in the time translation $b=t_{l}$ this discontinuity correlates with compressed wavelet and $\operatorname{CWT}\{f(t)\}(a, b)$ for these values of parameters a, b will be relatively high. It leads to precise location of the discontinuity in the signal. The mother wavelet functions Morlet is used in the presented CWT.

CWT decomposes (localizes) this function of signals in time-scale domain $(b, a) \subset R \times R$ and the area of time-scale windows is always constant and independent of the parameters a, b. In contrast to the Short-time Fourier transformation, the time-scale window changes its shape depending on the scale a. When displaying, we must remember that the parameter $a$ is inversely proportional to frequency, i.e. with the increase of the scale we have lower frequencies. The scalogram $\operatorname{SCAL}(b, a)$ is used to present CWT graphically. It is the function in two variables identified as square of modulus of the CWT.

$$
\operatorname{SCAL}(b, a)=|\operatorname{CWT}\{f(t)\}(a, b)|^{2} \text {. }
$$

\section{Experimental measurement and analysis of results}

The measured data presented in the article were acquired by the transducer which measures acceleration of the bridge in the middle of the span. It is labelled as BK1 and it was positioned on the surface of the side-walk (Fig. 1). The responses were measured while the vehicle was moving through the bridge with the constant speed. The moments when the vehicle comes to the bridge and when it leaves were detected through the steel plates with accelerometers fastened on them (Fig. 2).

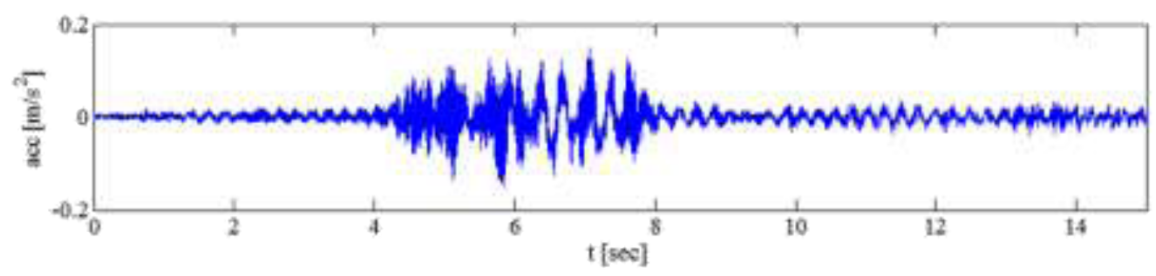

Fig. 3. The measured signal BK1 without the obstacle on the bridge. 
The analysed signals were measured for two cases. In the first case the vehicle moves with the speed $9.1 \mathrm{~ms}^{-1}$. There is no obstacle on the bridge. In the second case, the obstacle is positioned in the middle of the span to increase the excitation of the bridge. The obstacle is made of wood with special half-sine wave shape that is recommended by STN 736209 standard. The measured amplitude of acceleration on the bridge increases from $0.2 \mathrm{~ms}^{-2}$ to $1.2 \mathrm{~ms}^{-2}$. This significant increase in the response of the bridge is achieved by the obstacle. The speed of the vehicle $7.1 \mathrm{~ms}^{-1}$ is a little bit smaller than in the previous case. It is influenced with the fact that the driver of the vehicle intuitively slow down in front of the obstacle. This small difference in the speed is not considered in the analysis of the measured data as something that can dramatically change the results.

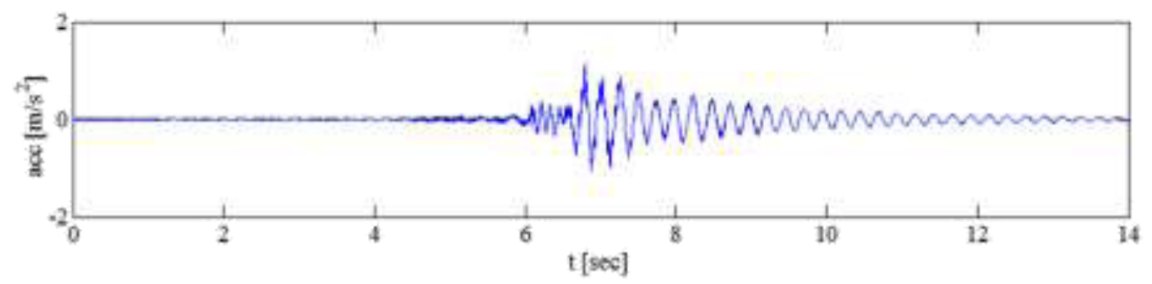

Fig. 4. The measured signal BK1 with the obstacle on the bridge.

The two measured signals are analysed with the two techniques of time-frequency analysis. Firstly, the STFT was used to analyse the changes in the frequency spectrum during the time. The Gaussian window with the length of 256 samples is used. The sample rate of the signal is $1000 \mathrm{~Hz}$ so the range of investigated frequency is from 0 to $500 \mathrm{~Hz}$. The frequency step in the results is $3.91 \mathrm{~Hz}$ with regards to the length of window (Fig. 5).

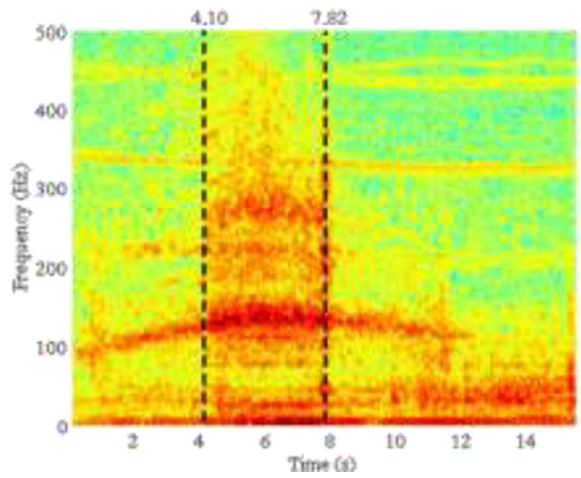

a) Response of the bridge without the obstacle

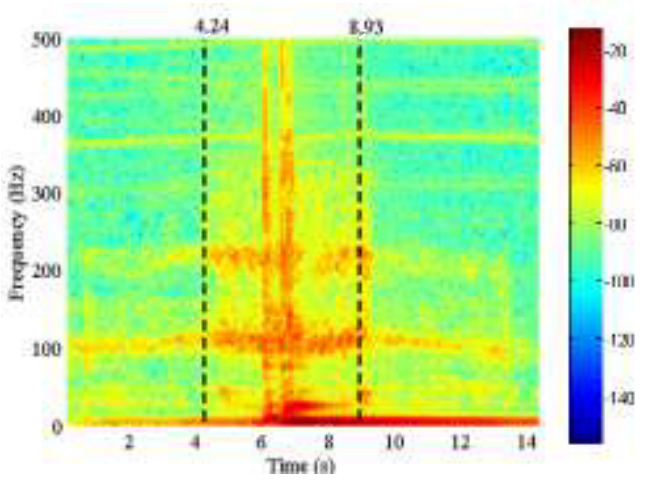

b) Response of the bridge with the obstacle

Fig. 5. Spectrogram of acceleration amplitude in the middle of the bridge (Gaussian window with length 256 samples).

The moments in time that were detected with steel plates when the vehicle came to their location are used to show how the STFT changes when the vehicle is on the bridge and when it is not. The amplitudes arises for the whole range of investigated frequency domain for both cases if the vehicle is on the bridge. There is possible to recognise the time when it starts very clearly. It means that this moment when the vehicle comes to the bridge is possible to detect. But the moment when the vehicle leaves the bridge is not so apparent because the bridge 
continues to oscillate with higher amplitudes even after the vehicle is not on the bridge. This time moment would be better identified if the damping characteristics of the bridge were considered but this has been not done till now.

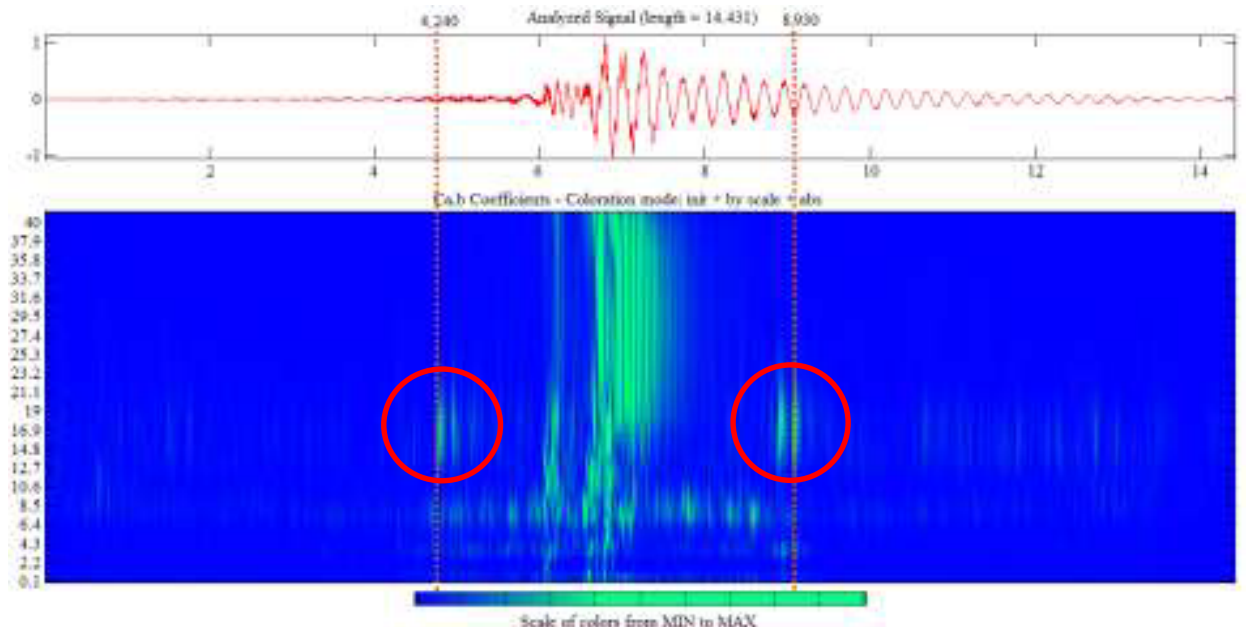

Fig. 6. Wavelet transform of acceleration in the middle of the bridge, Morlet wavelet, with the highlighted discontinuities.

The first one when there is an obstacle on the bridge there are detected the discontinuities in the signal in time when the vehicle comes and lives the bridge (Fig. 6). They can be used to recognise this time moment but it is not clear which is the source of these discontinuities in the signal. There is supposed that the steel plates are a source of these discontinuities as they are not fasten on the surface of the pavement and they can vibrate freely. They also produce a noise when the wheels moves through them during the measurement. However, there is no proof which could be used to confirm it.
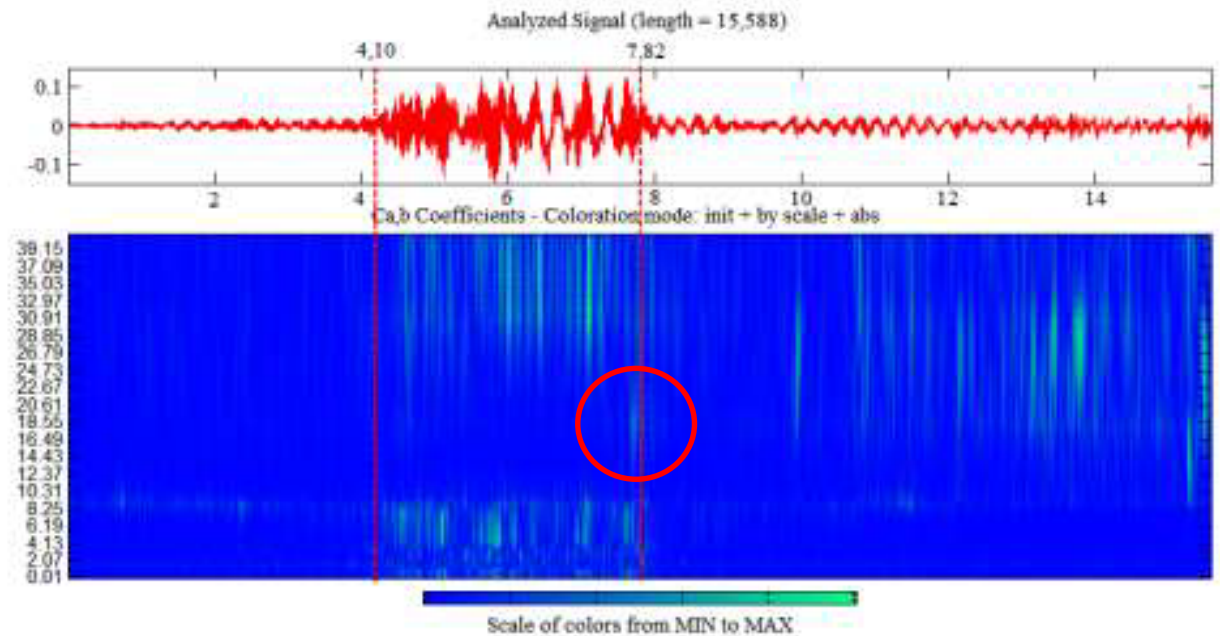

Fig. 7. Wavelet transform of acceleration in the middle of the bridge without obstacle, Morlet wavelet, with the highlighted discontinuities. 
The next approach focused on the identification of the time period when the vehicle is on the bridge is based on the CWT with the application of the Morlet (mother) wavelet. There are again investigated both cases with and without the obstacle on the bridge. The signal which was measured for the case when the obstacle is not on the bridge does not contain so big sources of discontinuities in the signal (Fig. 7). It is evident as there is not an obstacle. There is also a problem to identify the time period when the vehicle is on the bridge. In the moment when the vehicle comes to the bridge, there are not identified any noticeable discontinuities. Surprisingly, they are identified in this time moment in the previous case. There is identified the discontinuity only at the moment when the vehicle leaves the bridge.

\section{Conclusions}

The response of the bridge to the moving vehicle was measured by the accelerometer. The common techniques of time-frequency analysis of measured signals are used to recognise the time period in the signal when the vehicle is on the bridge. The results are compared with the identification of the moment when the vehicle comes to the bridge and when it leaves by steel plates positioned on the road of the bridge. It shows that both transformation from time domain to the frequency domain presented in the article can be used for this purpose. The STFT shows differences in the signal in time better for higher frequencies. Thanks this it can be detect the presence of the vehicle on the bridge. In the case, when there is an obstacle, it is also possible to detect the moment when the wheels bump to this obstacle. The CWT does not give so good results, but it could be better if the other type of mother wavelet were used. So the next investigation of this transformation should be focused on application of other types of wavelets to show if it can improve the results.

This paper was supported by the Grant National Agency VEGA of the Slovak Republic (grant No. 1/0005/16) and by the Grant Research Centre of The University of Zilina (grant No. 26220220183)

\section{References}

1. Nuno Manuel Mendes Maia, Julio Marins Manalvaou a Silva, Theoretical and experimental modal analysis (Research Studies Press, 1997)

2. Peeters Bart, C. E. Ventura, Mech. Syst. Signal Pr. 17, 5, 965-988 (2003)

3. A. Tesar, J. Melcer, Int. J. Numer. Meth. Eng. 74, 11, 1670-1678 (2008)

4. Yhong Hong Yan, Anyaho Miyamato, Zhongwei Jiang, Mech. Syst. Signal Pr. 23, 14741489 (2009)

5. Daniel Cantero, Mahir Ülker-Kaustell, Raid Karoumi, Mech. Syst. Signal Pr. 76, 518530 (2016)

6. K. Nayzar, Základy teorie waveletů (Univerzita Karlova v Praze, Karolinum, 2004)

7. D. Lee Fugal, Conceptual Wavelets in digital signal processing (Space \& Signals Technologies LLC, 2009) 